\title{
Modelling the feasibility of nutritional recommendations at the individual level
}

\author{
M. Maillot ${ }^{1}$, F. Vieux ${ }^{1}$, E. Ferguson ${ }^{2}$, M. J. Amiot-Carlin ${ }^{1}$, J. L. Volatier ${ }^{3}$ and N. Darmon ${ }^{1}$ \\ ${ }^{1}$ UMR Inserm 476/INRA 1260, Marseille, France, ${ }^{2}$ University of Otago, Dunedin, New Zealand \\ and ${ }^{3}$ AFSSA, Maisons-Alfort, France
}

The aim of the present study was to develop a method to evaluate the feasibility of modifying individual diets to achieve the French nutritional recommendations $^{(1)}$ (estimated average requirement; EAR) and to identify dietary characteristics that increase the likelihood of achieving them.

Dietary data from 1330 French adults participating in the French national INCA1 study ${ }^{(2)}$ (1999) were used. Starting from each observed diet, a linear programming model was used to assess whether it was possible to design a nutritionally-adequate diet using foods from the food repertoire of that individual (i.e. foods listed in their $7 \mathrm{~d}$ diet record). For each optimised diet its energy content was equal to the observed intake and its total weight was limited to $115 \%$ of its observed weight. Maximum weights were also imposed on individual foods, food groups and food subgroups. The constraints on each nutrient depended on its observed intake level (at least the EAR for observed intakes lower than the EAR; at least the RDA for observed intakes greater than the RDA; at least the observed intake when the observed intake was between the EAR and the RDA). In each optimised diet total departure from the observed amounts of each food consumed was minimised. Optimised diets that fulfilled all these constraints were categorised as feasible; those not fulfilling at least one constraint were categorised as not feasible. Dietary characteristics predicting diet feasibility were assessed by logistic regression, adjusting for total energy intake, gender and age. Specific dietary characteristics examined were the observed energy density (ED; kJ/kg), the mean adequacy ratio (MAR; \% nutrient adequacy) and the number of foods in the food repertoire. Diet modelling and statistical analysis were performed using SAS software (SAS Institute Inc., Cary NC, USA).

The number of foods in the observed diets ranged from fifteen to seventy-six, with an average of forty-five. Only $49 \%$ of the optimised diets were feasible, indicating that for $51 \%$ of diets it was not mathematically possible to achieve the EAR via realistic changes in the quantity of individual foods consumed. The dietary characteristics associated with achievement of the EAR were the number of foods in the food repertoire (OR 1.12) and the energy density (OR 0.84) and MAR (OR 1.35) of the diet.

For half the participants it was not possible to fulfil the nutritional recommendations without expanding their food repertoire. Nutrient density, energy density and food diversity were found to independently influence the feasibility of achieving them.

1. Martin A (editor) (2001) Apports Nutritionnels Conseillés pour la Population Française (Nutritional Advice for the French Population). Paris: Lavoisier.

2. Volatier J-L (2000) Enquête Individuelle et Nationale sur les Consommations Alimentaires (Individual and National Investigation into Food Consumption). Paris: Lavoisier. 